\title{
Torsion as Alternative to Curvature in the Description of Gravitation
}

\author{
V. C. de Andrade* \\ Universidade de Brasília \\ Brasília DF, Brazil \\ E-mail: andradedfis.unb.br
}

H. I. Arcos ${ }^{\dagger}$ and J. G. Pereira

Instituto de Física Teórica, UNESP

São Paulo SP, Brazil

E-mail: hiarcosdift.unesp.br, jpereiradift.unesp.br

\begin{abstract}
The general covariance principle, seen as an active version of the principle of equivalence, is used to study the gravitational coupling prescription in the presence of curvature and torsion. It is concluded that the coupling prescription determined by this principle is always equivalent with the corresponding prescription of general relativity. An application to the case of a Dirac spinor is made.
\end{abstract}

Fourth International Winter Conference on Mathematical Methods in Physics 09 - 13 August 2004

Centro Brasileiro de Pesquisas Fisicas (CBPF/MCT), Rio de Janeiro, Brazil

\footnotetext{
* Speaker.

${ }^{\dagger}$ Permanent address: Universidad Tecnológica de Pereira, A.A. 97, La Julita, Pereira, Colombia.
} 


\section{Introduction}

According to the teleparallel equivalent of general relativity, curvature and torsion are alternative ways of describing the gravitational field, and consequently related to the same degrees of freedom of gravity. However, more general gravity theories [1], like for example Einstein-Cartan and gauge theories for the Poincaré and the affine groups [2], consider curvature and torsion as representing independent degrees of freedom. In these models, differently from teleparallel gravity, torsion becomes relevant only when spins are important [3]. According to this point of view, torsion represents additional degrees of freedom in relation to curvature, and consequently new physics phenomena might be associated with it [4].

The above described difference rises a conceptual question on the actual role played by torsion in the description of the gravitational interaction. This question shows up due to the difficulty in determining the correct form of the gravitational coupling prescription in the presence of curvature and torsion. In fact, differently from all other interactions of nature, where the requirement of covariance does determine the gauge connection, in the presence of curvature and torsion, covariance — seen as a consequence of the strong equivalence principle [5]—is not able to determine the form of the gravitational coupling prescription. The reason for this indefiniteness is that the space of Lorentz connections is an affine space [6], and consequently one can always add a tensor (actually a Lorentz-valued covector) to a given connection without destroying the covariance of the theory. Notice that in the specific cases of general relativity and teleparallel gravity, characterized respectively by a vanishing torsion and a vanishing curvature, the above indefiniteness is absent since in these cases the connections are uniquely determined — and the corresponding coupling prescriptions completely specified — by the combined use of covariance and the strong equivalence principle. Notice furthermore that in the case of internal (Yang-Mills) gauge theories, where the concept of torsion is absent ${ }^{1}$, the above indefiniteness is not present either.

A possible answer to this problem can be obtained by using the so called principle of general covariance, an active version of the strong equivalence principle [8]. According to this principle, in order to make an equation generally covariant, a connection is always necessary, which is in principle concerned only with the inertial properties of the coordinate system under consideration. Then, by using the equivalence between inertial and gravitational effects, instead of representing inertial properties, this connection can equivalently be assumed to represent a true gravitational field. In this way, equations valid in the presence of gravitation are obtained from the corresponding special relativity equations. It is important to remark that general covariance by itself is empty of physical content as any equation can be made generally covariant. Only when use is made of the strong equivalence principle, and the inertial compensating term is interpreted as representing a true gravitational field, the principle of general covariance can be seen as an alternative version of the strong equivalence principle. Now, when the purely inertial connection is replaced by a connection representing a true gravitational field, the principle of general covariance naturally defines a covariant derivative, and consequently also a gravitational coupling prescription. The purpose of the present work will be to use this principle to determine the form of the gravitational coupling prescription in the presence of both curvature and torsion.

\footnotetext{
${ }^{1}$ We remark that absence of torsion, like in internal gauge theories, is different from the presence of a vanishing torsion, which happens in general relativity [7].
} 


\section{General covariance principle and coupling prescription}

The process of obtaining a gravitational coupling prescription from the general covariance principle comprises two steps. The first is to pass to a general nonholonomic frame, where inertial effects-which appear in the form of a connection, or compensating term-are present. Then, by using the strong equivalence principle, instead of inertial effects, the compensating term can be replaced by a connection representing a true gravitational field. In this way, a covariant derivative, and consequently a gravitational coupling prescription, is obtained. Let us then see how the whole process works.

\subsection{General frames}

Let us consider the Minkowski spacetime ${ }^{2}$ of special relativity, endowed with the Lorentzian metric $\eta$. In this spacetime one can take the frame $\delta_{a}=\delta_{a}{ }^{\mu} \partial_{\mu}$ as being a trivial (holonomous) tetrad, with components $\delta_{a}{ }^{\mu}$. Consider now a local, that is, point-dependent Lorentz transformation $\Lambda_{a}{ }^{b}=\Lambda_{a}{ }^{b}(x)$. It yields the new frame $h_{a}=h_{a}{ }^{\mu} \partial_{\mu}$, with components $h_{a}{ }^{\mu} \equiv h_{a}{ }^{\mu}(x)$ given by

$$
h_{a}^{\mu}=\Lambda_{a}^{b} \delta_{b}^{\mu}
$$

Notice that, on account of the locality of the Lorentz transformation, the new frame $h_{a}$ is nonholonomous, $\left[h_{b}, h_{c}\right]=f^{a}{ }_{b c} h_{a}$, with the coefficient of nonholonomy given by

$$
f_{b c}^{a}=h_{b}{ }^{\mu} h_{c}{ }^{\nu}\left(\partial_{\nu} h_{\mu}^{a}-\partial_{\mu} h^{a}{ }_{\nu}\right)
$$

Making use of the orthogonality property of the tetrads, therefore, we see from Eq. (2.1) that the Lorentz group element can be written in the form $\Lambda_{b}{ }^{d}=h_{b}{ }^{\rho} \delta_{\rho}{ }^{d}$. From this expression, it follows that

$$
\Lambda_{d}^{c}\left(h_{a} \Lambda_{b}{ }^{d}\right)=\frac{1}{2}\left(f_{b}^{c}{ }_{a}+f_{a}^{c}{ }_{b}-f_{b a}^{c}\right) .
$$

Let us consider now a vector field $v^{c}$ in the Minkowski spacetime. Its ordinary derivative in the frame $\delta_{a}$ is

$$
\partial_{a} v^{c}=\delta_{a}{ }^{\mu} \partial_{\mu} v^{c}
$$

Under a local Lorentz transformation, the vector field transforms according to $V^{d}=\Lambda^{d}{ }_{c} v^{c}$, and it is easy to see that $\partial_{a} v^{c}=\Lambda^{b}{ }_{a} \Lambda_{d}{ }^{c} \mathcal{D}_{b} V^{d}$, where

$$
\mathcal{D}_{a} V^{c}=h_{a} V^{c}+\Lambda_{d}^{c}\left(h_{a} \Lambda_{b}{ }^{d}\right) V^{b} .
$$

In the frame $h_{a}$, therefore, using the identity (2.3), the derivative (2.5) acquires the form

$$
\mathcal{D}_{a} V^{c}=h_{a} V^{c}+\frac{1}{2}\left(f_{b}{ }^{c} a+f_{a}^{c}{ }_{b}-f_{b a}^{c}\right) V^{b} .
$$

The freedom to choose any tetrad $\left\{h_{a}\right\}$ as a moving frame in the Minkowski spacetime introduces the compensating term $\frac{1}{2}\left(f_{b}{ }^{c} a+f_{a}{ }^{c}{ }_{b}-f^{c}{ }_{b a}\right)$ in the derivative of the vector field. This term, of course, is concerned only with the inertial properties of the frame.

\footnotetext{
${ }^{2}$ We use the Greek alphabet $\mu, \nu, \rho, \ldots=0,1,2,3$ to denote spacetime indices, and the Latin alphabet $a, b, c, \ldots=$ $0,1,2,3$ to denote indices related to the (local) tangent Minkowski spaces.
} 


\subsection{Equivalence between inertia and gravitation: gravitational coupling prescription}

According to the general covariance principle, the derivative valid in the presence of gravitation can be obtained from the corresponding Minkowski covariant derivative by replacing the inertial compensating term by a connection $A^{c}{ }_{a b}$ representing a true gravitational field. Considering a general Lorentz-valued connection presenting both curvature and torsion, one can always write [9]

$$
A_{b a}^{c}-A_{a b}^{c}=T_{a b}^{c}+f_{a b}^{c},
$$

with $T_{b a}^{c}$ the torsion of the connection $A^{c}{ }_{a b}$. Use of this equation for three different combination of indices gives

$$
\frac{1}{2}\left(f_{b}^{c}{ }_{a}+f_{a b}^{c}{ }^{c}-f_{b a}^{c}\right)=A_{a b}^{c}-K_{a b}^{c} .
$$

where

$$
K^{c}{ }_{a b}=\frac{1}{2}\left(T_{b}{ }^{c} a+T_{a}^{c} b-T^{c}{ }_{b a}\right)
$$

is the contortion tensor. Equation (2.8) is completely general, and is the crucial point of the approach. It is actually an expression of the equivalence principle in the sense that, whereas its left-hand side involves only inertial properties of the frames, its right-hand side contains purely gravitational quantities. Using this expression, the derivative (2.6) becomes

$$
\mathcal{D}_{a} V^{c}=h_{a} V^{c}+\left(A^{c}{ }_{a b}-K^{c}{ }_{a b}\right) V^{b} \equiv h_{a}{ }^{\mu} \mathcal{D}_{\mu} V^{c}
$$

where

$$
\mathcal{D}_{\mu} V^{c}=\partial_{\mu} V^{c}+\left(A_{a \mu}^{c}-K_{a \mu}^{c}\right) V^{a}
$$

is a generalized Fock-Ivanenko derivative. Using then the vector representation $\left(S_{a b}\right)^{c}{ }_{d}$ of the Lorentz generators [10], the generalized Fock- Ivanenko derivative (2.11) can be written in the form

$$
\mathcal{D}_{\mu} X^{c}=\partial_{\mu} X^{c}-\frac{i}{2}\left(A^{a b}{ }_{\mu}-K^{a b}{ }_{\mu}\right)\left(S_{a b}\right)^{c}{ }_{d} X^{d} .
$$

Now, although obtained in the case of a Lorentz vector field, the compensating term (2.3) can be easily verified to be the same for any field. In fact, denoting by $U \equiv U(\Lambda)$ the element of the Lorentz group in an arbitrary representation, it can be shown that [11]

$$
\left(h_{a} U\right) U^{-1}=-\frac{i}{4}\left(f_{b c a}+f_{a c b}-f_{c b a}\right) J^{b c},
$$

with $J^{b c}$ denoting the corresponding Lorentz generator. In the case of fields carrying an arbitrary representation of the Lorentz group, therefore, the covariant derivative (2.12) acquires the form

$$
\mathcal{D}_{\mu}=\partial_{\mu}-\frac{i}{2}\left(A^{a b}{ }_{\mu}-K^{a b}{ }_{\mu}\right) J_{a b}
$$

This means that, in the presence of curvature and torsion, the gravitational coupling prescription implied by the general covariance principle amounts to replace

$$
\partial_{a} \equiv \delta^{\mu}{ }_{a} \partial_{\mu} \rightarrow \mathcal{D}_{a} \equiv h^{\mu}{ }_{a} \mathcal{D}_{\mu}
$$

We notice finally that, due to the relation

$$
A^{a b}{ }_{\mu}-K_{\mu}^{a b}=\stackrel{\circ}{A b}_{\mu},
$$

with $\stackrel{\circ}{A b}_{\mu}$ the spin connection of general relativity, the above coupling prescription is clearly equivalent with the coupling prescription of general relativity. 


\section{Example: the spinor field}

The gravitational coupling prescription (2.14-2.15) has already been applied to study the motion of both a spinless and a spinning particle [12]. Here, we apply it to the case of a Dirac spinor in the presence of curvature and torsion.

\subsection{Dirac equation}

The Dirac equation in flat spacetime can be obtained from the Lagrangian (we use units in which $\hbar=c=1$ )

$$
\mathcal{L}=\frac{i}{2}\left(\bar{\psi} \gamma^{a} \delta_{a}{ }^{\mu} \partial_{\mu} \psi-\partial_{\mu} \bar{\psi} \gamma^{a} \delta_{a}{ }^{\mu} \psi\right)-m \bar{\psi} \psi
$$

where $\delta_{a}{ }^{\mu}$ is a trivial tetrad, $m$ is the particle's mass, and $\left\{\gamma^{a}\right\}$ are (constant) Dirac matrices in a given representation. Making use of the coupling prescription (2.14-2.15), with $J^{b c}=\sigma^{b c} / 2:=$ $(i / 4)\left[\gamma^{b}, \gamma^{c}\right]$ the spinor representation of the Lorentz generators, we obtain

$$
\mathcal{L}=\frac{i}{2}\left(\bar{\psi} h_{a}{ }^{\mu} \gamma^{a} \mathcal{D}_{\mu} \psi-\mathcal{D}_{\mu} \bar{\psi} h_{a}{ }^{\mu} \gamma^{a} \psi\right)-m \bar{\psi} \psi,
$$

where the Fock-Ivanenko derivative operator is given by

$$
\mathcal{D}_{\mu} \psi=\partial_{\mu} \psi-\frac{i}{4}\left(A^{b c}{ }_{\mu}-K^{b c}{ }_{\mu}\right) \sigma_{b c} \psi
$$

This covariant derivative yields the coupling prescription for spin-1/2 fields in the presence of curvature and torsion. As usual, a functional derivative with respect to $A^{b c}{ }_{\mu}-K^{b c}{ }_{\mu} \equiv A^{b c}{ }_{\mu}$ yields the spin tensor. A straightforward calculation shows that the Dirac Lagrangian (3.2) gives rise to

$$
i \gamma^{a} h_{a}{ }^{\mu} \mathcal{D}_{\mu} \psi=m \psi
$$

which is the Dirac equation in the presence of curvature and torsion. Of course, as already mentioned, it is equivalent with the Dirac equation in the context of general relativity [13].

\subsection{Torsion decomposition}

As is well known, torsion can be decomposed in irreducible components under the global Lorentz group [14]

$$
T_{\lambda \mu v}=\frac{2}{3}\left(t_{\lambda \mu v}-t_{\lambda v \mu}\right)+\frac{1}{3}\left(g_{\lambda \mu} T_{v}-g_{\lambda v} T_{\mu}\right)+\varepsilon_{\lambda \mu \nu \rho} S^{\rho} .
$$

In this expression, $T_{\mu}$ and $S^{\rho}$ represent the vector and axial parts of torsion, defined respectively by

$$
T_{\mu}=T_{\nu \mu}^{v} \quad \text { and } \quad S^{\mu}=\frac{1}{6} \varepsilon^{\mu v \rho \sigma} T_{v \rho \sigma}
$$

and $t_{\lambda \mu \nu}$ is the purely tensor part, which satisfies the properties $t_{\lambda \mu \nu}=t_{\mu \lambda v}$ and $t_{\mu \lambda}^{\mu}=0=t_{\lambda \mu}^{\mu}$. As a simple calculation shows,

$$
\frac{i}{4} K_{a}^{b c} \gamma^{a} \sigma_{b c}=-\gamma^{a}\left(\frac{1}{2} T_{a}+\frac{3 i}{4} S_{a} \gamma^{5}\right)
$$

with $\gamma^{5}=\gamma_{5}:=i \gamma^{0} \gamma^{1} \gamma^{2} \gamma^{3}$. The covariant derivative (3.3) then becomes

$$
\mathcal{D}_{\mu} \psi=\left(\partial_{\mu}-\frac{i}{4} A^{b c}{ }_{\mu} \sigma_{b c}-\frac{1}{2} T_{\mu}-\frac{3 i}{4} S_{\mu} \gamma^{5}\right) \psi .
$$


We observe that, whereas the functional derivative of the Lagrangian (3.2) in relation to the connection $A^{b c}{ }_{\mu}$ still gives the spin tensor, derivatives with respect to $T_{\mu}$ and $S_{\mu}$ give respectively the vector and the axial-vector currents of the spinor field.

Substituting now the covariant derivative (3.8) in the equation (3.4), we get

$$
i \gamma^{\mu}\left(\partial_{\mu}-\frac{i}{4} A_{\mu}^{b c} \sigma_{b c}-\frac{1}{2} T_{\mu}-\frac{3 i}{4} S_{\mu} \gamma^{5}\right) \psi=m \psi
$$

where $\gamma^{\mu} \equiv \gamma^{\mu}(x)=\gamma^{a} h_{a}{ }^{\mu}$. This is the Dirac equation in the presence of curvature and torsion, written in terms of irreducible components for torsion. In the specific case of teleparallel gravity, $A^{b c}{ }_{\mu}=0$, and the resulting Dirac equation turns out to be written in terms of the vector and axialvector torsions only [11]. We remark that in the general relativity case, where the Fock-Ivanenko

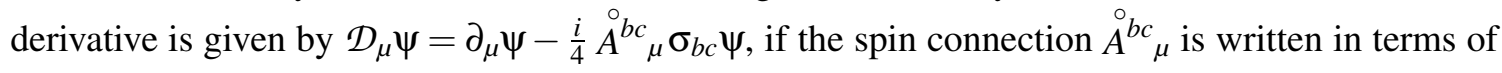
the coefficient of nonholonomy $f_{b c}^{a}$, a decomposition similar to (3.7) can be made, and the Dirac equation turns out to be written in terms of the trace and the pseudo-trace of $f^{a}{ }_{b c}$ only ${ }^{3}$.

\section{Final comments}

A fundamental difference between general relativity and teleparallel gravity is that, whereas in the former curvature is used to geometrize the gravitational interaction-spinless particles follow geodesics - in the latter torsion describes the gravitational interaction by acting as a forcetrajectories are not given by geodesics, but by force equations [15]. According to the teleparallel approach, therefore, the role played by torsion is quite well defined: it appears as an alternative to curvature in the description of the gravitational field, and is consequently related with the same degrees of freedom of gravity. Now, this interpretation is completely different from that appearing in more general theories, like Einstein-Cartan and gauge theories for the Poincaré and the affine groups. In these theories, curvature and torsion are considered as independent fields, related with different degrees of freedom of gravity, and consequently with different physical phenomena. This is a conflicting situation as these two interpretations cannot be both correct.

As an attempt to solve the above described paradox, we have used the general covariance principle - seen as an alternative version of the strong equivalence principle - to study the gravitational coupling prescription in the presence of curvature and torsion. According to this principle, the dynamical spin connection, that is, the spin connection defining the covariant derivative, and consequently the gravitational coupling prescription, is $A_{a b}^{c}-K_{a b}^{c}$. Even in the presence of curvature and torsion, therefore, torsion appears as playing the role of gravitational force. This result gives support to the point of view of teleparallel gravity, according to which torsion does not represent additional degrees of freedom of gravity, but simply an alternative way of representing the gravitational field. Furthermore, since $A_{a b}^{c}-K_{a b}^{c}={\stackrel{\circ}{A^{c}}}_{a b}$, the ensuing coupling prescription will always be equivalent with the coupling prescription of general relativity, a result that reinforces the completeness of this theory.

It is important to add that, at least up to now, there are no compelling experimental evidences for new physics associated with torsion. We could then say that the teleparallel point of view is favored by the available experimental data. For example, no new gravitational physics has ever

\footnotetext{
${ }^{3}$ We thank R. Aldrovandi for calling our attention to this point.
} 
been reported near a neutron star. On the other hand, it is true that, due to the weakness of the gravitational interaction, no experimental data exist on the coupling of the spin of the fundamental particles to gravitation. Anyway, precision experiments [16] either in laboratory or as astrophysical and cosmological tests are expected to be available in the foreseeable future, when then a final answer will hopefully be achieved.

\section{Acknowledgments}

The authors would like to thank R. Aldrovandi and R. A. Mosna for very useful discussions. They would like also to thank FINATEC (UnB), COLCIENCIAS (Colombia), CAPES, CNPq and FAPESP for partial financial support.

\section{References}

[1] M. Blagojević, Gravitation and Gauge Symmetries, IOP Publishing, Bristol 2002.

[2] F. W. Hehl et al, Metric Affine Gauge Theory of Gravity: Field Equations, Noether Identities, World Spinors and Breaking of Dilation Invariance, Phys. Rep. 258 (1995) 1.

[3] F. Gronwald and F. W. Hehl, On the Gauge Aspects of Gravity, in Proceedings of the 14th School of Cosmology and Gravitation, Erice, Italy, World Scientific, Singapore 1996.

[4] See, for example, I. L. Shapiro, Physical Aspects of the Space-time Torsion, Phys. Rep. 357 (2002) 113.

[5] S. Weinberg, Gravitation and Cosmology, Wiley, New York 1972.

[6] S. Kobayashi and K. Nomizu, Foundations of Differential Geometry, Vol. 1, Wiley, New York 1963.

[7] R. Aldrovandi and J. G. Pereira, An Introduction to Geometrical Physics, World Scientific, Singapore 1995.

[8] H. I. Arcos and J. G. Pereira, Torsion and the Gravitational Interaction, Class. Quant. Grav. 21 (2004) 5193 [gr-qC/ 0408096$]$.

[9] R. Aldrovandi, P. B. Barros and J. G. Pereira, Gravitation and Anholonomy, Gen. Rel. Grav. 35 (2003) 991 [gr-qc/0301077].

[10] P. Ramond, Field Theory: A Modern Primer, 2nd edn, Addison-Wesley, Redwood 1989.

[11] R. A. Mosna and J. G. Pereira, Some Remarks on the Coupling Prescription of Teleparallel Gravity, Gen. Rel. Grav. 36 (2004) 2525 [gr-qc/ 0312093 ].

[12] H. I. Arcos, V. C. de Andrade and J. G. Pereira, Torsion and Gravitation: A New View, Int. J. Mod. Phys. D13 (2004) 807 [gr-qc/ 0403074 ].

[13] P. A. M. Dirac, The Electron Wave Equation in Riemann Space, in Planck Festschrift, ed. W. Frank, Deutscher Verlag der Wissenschaften, Berlin 1958.

[14] K. Hayashi and T. Shirafuji, New General Relativity, Phys. Rev. D19 (1979) 3524.

[15] V. C. de Andrade and J. G. Pereira, Gravitational Lorentz Force and the Description of the Gravitational Interaction, Phys. Rev. D56 (1997) 4689 [gr-qc/ 9703059 ].

[16] R. T. Hammond, Torsion Gravity, Rep. Prog. Phys. 65 (2002) 599. 